\title{
The Countermeasure Research on Development of Hainan Translation Services Based on Construction of International Tourism Island
}

\author{
Changqing $\mathrm{Fu}$ \\ Hainan Foreign Language College of Professional Education, Wenchang, 571321, China
}

\begin{abstract}
Keywords: International tourism island construction; Hainan translation service industry; Development bottleneck; Countermeasure study
\end{abstract}

\begin{abstract}
As international tourism island rapidly advances, Hainan tourism ushers in satisfactory development trend. Annual output value of tourism increases sharply. This promotes harmonious development of Hainan economy. Hainan translation service industry based in international tourism island construction has unprecedented industry development opportunity. Meanwhile, it is also faced with numerous difficulties and problems. This paper mainly analyzes opportunity of translation service industry development from the importance of Hainan translation service industry in international tourism island, proposes countermeasures and suggestions, deeply explores existing problems and causes and puts forward targeted countermeasures and suggestions.
\end{abstract}

\section{Introduction}

Since the construction of Hainan International Tourism Island rises to national strategy, Hainan translation service industry development as international tourism island construction presents a prosperous scene. To help Hainan to become an international tourism island with world influence, Hainan translation service industry still has a long way to go. At present, although Hainan translation service industry is faced with a very rare development opportunity, development status of this industry is not optimistic. On the whole, Hainan translation service industry has cultivated a large group of professional translation talents for Hainan in the past years, but the increasing speed of translation talents cannot catch up with growth rate of whole translation market consumption. At present, overall development of Hainan translation service industry is characterized by disorder and vicious competitions. Thus, it is required to start from macroscopic development to formulate long-term development planning of Hainan translation service industry so as to help the whole industry develop in a healthier and more orderly way so as to meet the demand of Hainan International Tourism Island construction for high-quality professional translation talents.

\section{Importance of Hainan translation service industry in international tourism island construction}

Hainan translation service industry has gained great development opportunity under the background of Hainan International Tourism Island construction. Hainan provincial government has issued some local policies to drive rapid development of Hainan translation service industry. For example, cultural management department of Hainan government formulated three-year development planning for Hainan translation service industry in 2012. The primary cause for valuing development of translation service industry is that international tourism island needs more professional high-quality translation talents so as to meet expansion demand of international tourism, commerce and trade. Therefore, translation service industry occupies a very important position in Hainan International Tourism Island construction. Without sufficient translation talents, it is very difficult to achieve the goal of international tourism island construction. In particular, as some international large-scale conferences and competitions are held in Haikou, Sanya and Qionghai etc., such as Boao Forum for Asia and Miss World final, higher development requirements are proposed for Hainan translation service industry. Seeing from development status of Hainan translation service industry, although the number of employees in Hainan translation service industry has increased sharply in the past years, the urgent demand of Hainan International Tourism Island for translation 
talents cannot be met. There is badly short of some minority language translation talents, which event influences general situation of Hainan International Tourism Island construction. In 2013, a French enterprise planned to sign agricultural product purchase and sale contract with Hainan. However, since professional quality of French translators was poor, they could not clearly explain contract articles. This made French customers very dissatisfied. Finally, the contract was not signed. This caused a huge loss to local agricultural product export. During receiving international tourists, some translators have defects in translation skills. If no effective measures are taken to change laggard development of Hainan translation service industry, potential threat will be brought to Hainan International Tourism Island construction.

\section{Numerous development bottlenecks faced by Hainan translation service industry low \\ The number of talents in translation service industry is insufficient, and overall quality is}

Hainan translation service industry has gained significant development in international tourism island construction. The survey data show at present, the number of employees engaged in translation service in Hainan translation companies and tourism companies has exceeded 80000. More domestic and overseas translation talents swarm in Hainan to look for jobs. But the number of translation talents cannot satisfy huge consumption demand of Hainan translation service industry. Many translation companies have to spend higher remuneration to invite translation talents due to the shortage of talents. The actual effect is not obvious. Sometimes, vicious talent contest occurs among different translation companies. Take a famous translation company in Hainai - Yilin Translation Company for example. At present, the company now owns 326 professional translation talents and will undertake plentiful translation tasks. To make for talent shortage, the salary for translation talents has increased to annual salary of 1 million Yuan from over 100000 Yuan. However, actual recruitment effect is not obvious. More high-quality translation talents were not recruited. Instead, translation talents cultivated by the company left the company. Except the insufficient number of translation talents, overall translation quality of employees in Hainan translation service industry is not high. Especially, minority language translation talents are badly short. Some translation companies have to spend heavy money inviting employees from foreign translation companies in order to meet needs of minority language translation. However, in the long run, this is not the fundamental countermeasure of solving the problem.

\section{Translation service industry fails to synchronously develop construction of Hainan International Tourism Island}

The construction of Hainan International Tourism Island changes quickly, and the development speed is amazing. Obviously, the development of Hainan translation service industry is not synchronous with international tourism island construction, and even lags behind development of international tourism island construction. Seeing from current construction speed of Hainan International Tourism Island, Hainan translation service industry needs to develop at the speed of $30 \%$ annually in order to catch up with development pace of Hainan International Tourism Island construction. However, seeing from development status of Hainan translation service industry, the development speed of Hainan translation service industry is just $19.67 \%$. There is still a significant gap. Furthermore, as international tourism island construction continuously speeds up, such gap has the rising trend. Hainan translation service industry has realized severity of this problem, and taken active countermeasures. Relevant departments of Hainan government also have formulated and issued some policies and regulations to promote development of Hainan translation service industry. However, it will still take some time to give play to actual effects of these institutional measures. The development of Hainan translation service industry lags behind international tourism island construction. Such situation is difficult to change in a short term. Therefore, Hainan translation service industry has started to obviously lag behind construction speed of Hainan International Tourism Island. If no effective measures are taken, Hainan International Tourism Island construction 
will be faced with the plight of translation talent shortage, which will further influence realization of provincial construction goal.

\section{Translation service industry fails to establish orderly competition idea}

Seeing from development status of Hainan translation service industry, Hainan translation service industry fails to establish effective industry development norms. Overall translation service industry is in disorderly competition state. In short, Hainan translation service industry fails to establish effective competition idea. This, translation service market is disorderly. Some without translation service qualification are driven by high benefits to invest in setting up translation companies one after another. This makes overall industrial quality of Hainan translation service industry low. Some translation companies adopt unfair competition means in market competitions of translation service industry, which is also one of significant factors causing disorderly development of Hainan translation service industry. In March 2014, a large tourism company in Sanya translation strove for translation talents from local translation companies. Finally, two companies confronted at court, which led to serious loss to both parties and imposed negative effects on the whole industry. To establish orderly competition way and let all translation companies adopt fair competition means to participate in market competitions must be valued in development of translation service industry. In the future, if Hainan translation service industry does not pay attention to the above work, hidden danger will be buried for whole translation service industry and thus affect general situation of Hainan International Tourism Island construction.

\section{Hainan provincial government fails to fully support development of translation service industry}

Hainan provincial government lays particular emphasis on infrastructure support in international tourism island construction in terms of manpower, material resources and financial resources. Of course, it is very necessary to invest in hardware faculties. These are the priority among priorities in construction of Hainan International Tourism Island. It is inadvisable to ignore development of software industry - translation service industry. Laggard development of Hainan translation service industry is closely related to insufficient support force resulting from ignorance of policies by Hainan provincial government. Since Hainan International Tourism Island construction improved to international strategy in 2010, the whole Hainan Province actively devotes itself in international tourism island construction. Government investment plays commanding role in the construction. Early investment support idea which places particular emphasis on hardware infrastructure brings rapid improvement of hardware development of Hainan and meanwhile leads to sufficient software construction. In 2012, Hainan Province started to realize the importance of software facilities construction, such as putting forward some support strategies in development of translation service industry. On the whole, since translation service industry gained government support late, disconnection with development of other industries starts to appear. So far, the support policy of Hainan provincial government for translation service industry is not as meticulous and all-round as other meticulous facilities industry. Thus, development bottleneck problems of Hainan translation service industry are not relieved effectively.

\section{Countermeasures and suggestions on development of Hainan translation service industry}

\section{Introduce high-quality translation talents at home and abroad}

Hainan translation service industry should adopt more active measures to introduce translation talents around the world. Although there are many training institutions for translation talents in China, high-end translation talents mainly concentrate in developed metropolises or regions. There is still short of translation talents in underdeveloped area. Therefore, based on recruiting talents from Chinese famous colleges, talent recruitment range should expand to the world. YGYM Sanya Company has started to recruit translation talents from countries in Europe and America and has gained certain achievements. Such positive practice should be absorbed by other translation companies in Hainan. Especially for minority language translation talents, it is better to directly recruit related translation talents from corresponding countries so as to effectively relieve shortage of 
translation talents in Hainan and especially minority language translation talents. With the support of a large number of high-quality translation talents, Hainan translation service industry can offer comprehensive and effective translation service for international tourism island construction.

\section{Make efforts to achieve harmony of development of Hainan translation service industry and international tourism island construction}

International tourism island construction cannot be separated from development and support of translation service industry. Hence, Hainan translation service industry should speed up development pace and achieve synchronous development with international tourism island construction as soon as possible. The author suggests that Hainan translation service industry should refer to the practice of five-year development planning to establish five-year development plan of this industry. It is required to lay down overall planning of industrial development, establish macroscopic development idea of translation service industry and take effective measures in order to shorten the gap with international tourism island construction. The author considers the annual development speed of Hainan translation service industry should reach $35 \%$ in order to catch up with and satisfy the desire of Hainan International Tourism Island construction for translation talents. Meanwhile, development speed and improvement of development quality of translation service industry should be valued. If unilateral quantity development is pursued, and connotation quality does not improve, this will be to the disadvantage of long-term development of translation service industry finally.

\section{Establish orderly competition idea}

Seeing from translation service industry, orderly competition idea for the whole industry needs to be established. Numerous chaotic phenomena exist in the development of Hainan translation service industry. It is required to take effective measures to actively solve these chaotic phenomena. The author suggests provincial translation service association should be established to make the development of translation service industry on longer disorderly. In this way, more translation companies can be engaged in translation according to industry standards and avoid vicious competitions or mutual fraud as far as possible. To form such orderly competition idea, government and related departments carry out effective supervision. As for market disorder or fraud behaviors of translation service industry, they should be strictly standardized and supervised. Translation institutions disrupting market order should be severely punished. In one word, as long as Hainan translation service industry establishes positive and orderly competition idea, the development of Hainan translation service industry can be more orderly under powerful supervision of non-government management organization and culture department of government. This will offer more beneficial translation service support for Hainan International Tourism Island construction.

The government should increase support for development of translation service industry

The development of Hainan translation service industry cannot be separated form vigorous support of translation service industry. Hainan provincial government should pay more attention to steady development of translation service industry based on paying attention to hardware infrastructure construction in future international tourism island. In annual financial budget, the government should list special financial support funds for development of translation service industry. Meanwhile, it is required to support of translation service industry development in terms of personnel management and personnel management. Translation service industry should actively seek government's help, and drive the drive to formulate and issue more policies and regulations to protect and support development of this industry. In brief, the development of Hainan translation service industry cannot be separated from powerful help of government. Government's support of translation service industry development is not just beneficial to translation service industry development, but also provides more high-quality professional translation talents for Hainan International Tourism Island construction. This is a win-win event

\section{Conclusions}

To sum up, Hainan International Tourism Island construction needs a large number of professional translation talents. However, currently, the development of Hainan translation service industry 
obviously lags behind provincial construction and development pace, and translation service industry itself also has some defects. If such situation is not changed, this will be adverse to long-term development of translation service industry construction. As long as the government makes progress together with the industry, the development of Hainan translation service industry will certainly usher in exuberant spring.

\section{Acknowledgments}

This paper is one of the research results produced from the 2015 Planned Research Project of Hainan Provincial Philosophy and Social Sciences Titled The Countermeasure Research on Development of Hainan Translation Services Based on Construction of International Tourism Island, Project No.: HNSK(Z)12-53.

\section{References}

[1] Yan Biyu, cCurrent situation and development of Wenzhou translation service industry. China Business \& Trade, 2014 (14): 189-191.

[2] Li Xiang, Li Linping, Zhan Lili, Analysis of China Business \& Trade of translation companies. Jin Tian, 2013 (4): 343.

[3] Chu Dongwei, Study on international translation service trade and Chinese translation industry. International Economics and Trade Research, 2004 (2): 67-69.

[4] Li Yan, Wang Yongting, Yin Zhengjiang, International tourism island construction and cultural tourism development of Hainan. Enterprise Economy, 2011 (06): 159-162.

[5] Liu Jun, Perception and attitude of Hainan residents to policy influence of international tourism island. Tourism Tribune, 2011 (06): 21-28. 